OPEN ACCESS

Edited by:

Tuan Leng Tay,

University of Freiburg, Germany

Reviewed by:

Javier Ochoa-Reparaz,

Eastern Washington University,

United States

lain Comerford,

University of Adelaide, Australia

*Correspondence:

Jennifer L. Gommerman

jen.gommerman@utoronto.ca

Specialty section:

This article was submitted to

Multiple Sclerosis

and Neuroimmunology,

a section of the journal

Frontiers in Immunology

Received: 15 July 2021

Accepted: 31 August 2021

Published: 15 September 2021

Citation:

Pu A, Lee DSW, Isho B,

Naouar I and Gommerman JL (2021)

The Impact of $\lg A$ and the

Microbiota on CNS Disease.

Front. Immunol. 12:742173.

doi: 10.3389/fimmu.2021.742173

\section{The Impact of IgA and the Microbiota on CNS Disease}

\author{
Annie Pu, Dennis S. W. Lee, Baweleta Isho, Ikbel Naouar and Jennifer L. Gommerman * \\ Department of Immunology, Faculty of Medicine, University of Toronto, Toronto, ON, Canada
}

Although anatomically distant from the central nervous system (CNS), gut-derived signals can dynamically regulate both peripheral immune cells and CNS-resident glial cells to modulate disease. Recent discoveries of specific microbial taxa and microbial derived metabolites that modulate neuroinflammation and neurodegeneration have provided mechanistic insight into how the gut may modulate the CNS. Furthermore, the participation of the gut in regulation of peripheral and CNS immune activity introduces a potential therapeutic target. This review addresses emerging literature on how the microbiome can affect glia and circulating lymphocytes in preclinical models of human CNS disease. Critically, this review also discusses how the host may in turn influence the microbiome, and how this may impact CNS homeostasis and disease, potentially through the production of IgA.

Keywords: IgA, gut microbiome, ageing, multiple sclerosis, neurodegeneration

\section{INTRODUCTION}

Complex diseases of the central nervous system (CNS) are caused by a combination of genetic and environmental factors. Human studies and animal models have demonstrated that commensal microbes residing in a host can influence CNS disease (Figure 1), adding additional complexity to unraveling the etiology of these diseases. While cheaper and more efficient sequencing technologies has facilitated the human microbiome project (24), we are only at the beginning of identifying disease $v s$. health-promoting microbes, the environmental and genetic factors that promote such communities, and the functional output of these communities.

Two main factors may modify microbiota. First, the microbiota is highly context-dependent and modulated by geographic location and diet (25). Strong evidence suggests diet-based alterations in the microbiome come from extreme diet changes (26) or adoption of new cultural dietary habits $(27,28)$. There is increasing appreciation for the role of diet-microbiome interactions in CNS diseases (29). Second, internal factors such as host genetics, age, and sex are also important determinants for selecting the gut microbiota (30).

Herein we review emerging literature linking host-microbiome interactions to lymphocytes and glial cells in the context of CNS diseases. We describe two key host factors, intestinal IgA and ageing, that have a profound impact on shaping the microbiome. We also provide examples for how these factors impact lymphocytes and glial cells in the context of CNS disease. In summary, we provide a working model for how interactions between host factors (IgA and ageing) contribute towards 


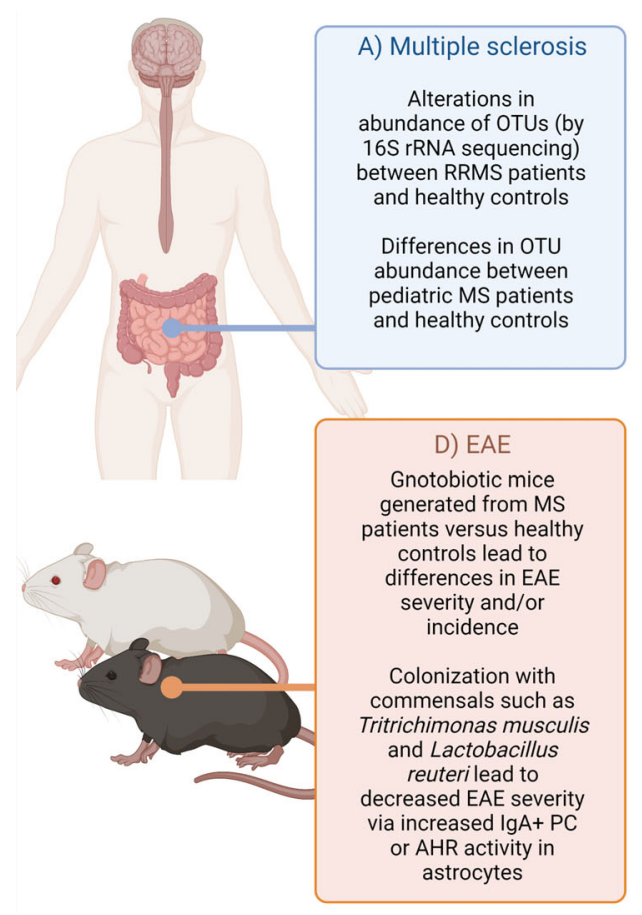

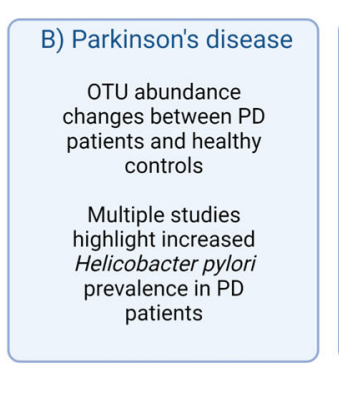

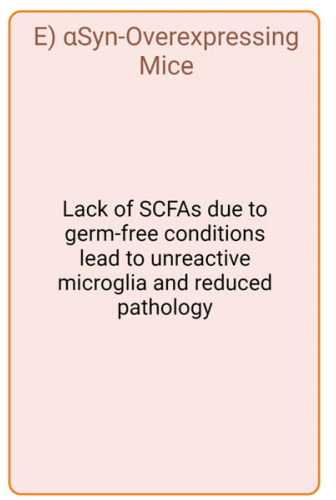

C) Alzheimer's disease

Patients with increased amyloid uptake were with SCFAs and positively associated with LPS

F) Alzheimer Models

Germ-free or probiotic interventions in mouse models that result in increased amyloid deposits in the brain can lead to pathological and cognitive improvements

FIGURE 1 | Highlighted evidence for the relationship of microbiota affects in CNS-specific human diseases and animal models. Complex diseases of the CNS are often difficult to query in humans due to scarcity of tissue samples. However, combining evidence from patients, healthy controls (A-C) (1-14), as well as animal models (D-F) (15-23) can provide some suggestive evidence on how the microbiota may impact disease. Figure made using (BioRender.com).

shaping the microbiome which in turn can influence lymphocyte and glial cell behavior in the context of CNS disease (Figure 2).

\section{INFLUENCE OF THE MICROBIOTA ON LYMPHOCYTES IN CNS DISEASE}

Correlative data in MS and mouse models demonstrate a bidirectional interaction between the gut and the CNS $(15,16$, 31-33); identifying specific contributions of the gut microbiome to CNS disease is imperative for understanding disease pathogenesis. While aberrantly activated lymphocytes are a hallmark of multiple sclerosis (MS), this is less studied in Alzheimer's and Parkinson's disease (AD, PD). Thus, this section will focus on the impact of microbiota on lymphocyte function in MS.

\section{T Lymphocytes}

Although alterations in the microbiome have been reported in MS case-control studies (34), testing causal associations between these alterations and disease risk requires animal models. Germfree (GF) mice lack commensal microbiota and thus present a "blank slate" for exploring the impact of commensal microbes on disease. GF mice fail to develop experimental autoimmune encephalomyelitis (EAE) (35), but when gnotobiotically recolonized or monocolonized with segmented filamentous bacteria (SFB), EAE is rescued. SFB enhances $\mathrm{T}_{\mathrm{H}} 17$ cell induction (36), $\mathrm{T}$ cells that are critical in causing pathology in EAE. Regulatory T cells (Tregs) are similarly sensitive to the gut microbiome. Their polarization from naïve $\mathrm{T}$ cells can be potentiated by Bacteroides fragilis polysaccharide in EAE (17, 35). Interestingly, following transplantation of human MS stool samples into mice (fecal microbiome transplant; FMT), several bacterial species were linked to alterations in $\mathrm{T}_{\mathrm{H}} 1$ and Treg differentiation, and consequently EAE phenotype $(37,38)$.

\section{B Lymphocytes}

$\mathrm{B}$ cells produce antibodies, present antigen to $\mathrm{T}$ cells, and secrete cytokines. When antigen binds to a $B$ cell receptor, these antigenspecific $B$ cells are activated and undergo somatic hypermutation and affinity maturation in germinal centers (GC) (39), generating high-affinity antigen-specific receptors. GC B cells can also classswitch to generate different antibody isotypes with specialized effector functions (IgA, IgE, IgG). Mature B cells can also differentiate into memory B cells or antibody-secreting cells (ASCs) (40). ASCs comprise both proliferative plasmablasts (PBs) and terminally differentiated plasma cells (PCs) $(39,41)$. Alterations in microbial abundance have been correlated with changes in regulatory B cell (Breg) induction $(18,42)$. Antibiotic treatment enhanced frequencies of IL-10-producing Bregs at baseline and in EAE (42). In addition, a role for gut-derived commensal-reactive IgA ${ }^{+}$ASCs in attenuating EAE and possibly also MS has been shown $(18,19)$, described below. 


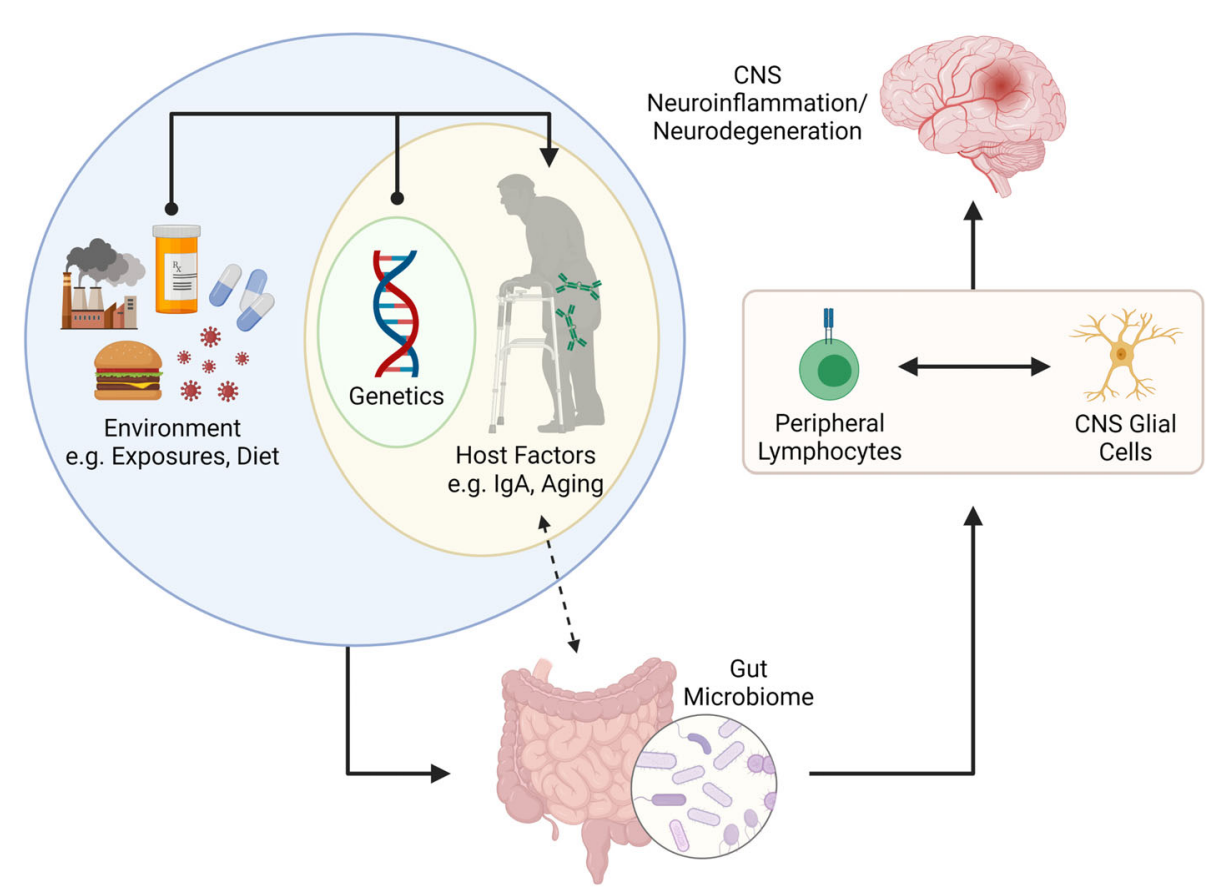

FIGURE 2 | Putative connection between the gut microbiome and CNS neuroinflammation and neurodegeneration. The gut microbiome is shaped by internal (e.g., genetics and other host factors such as mucosal IgA and age) and external factors (e.g., environmental exposures, infections, diet, etc.). Recent literature has suggested that IgA plays a key role in determining the microbes that reside in the gut, but that IgA levels can also be influenced by colonizing microbiota. The gut microbiome is important for programming of peripheral lymphocytes but can also impact the phenotype and function of CNS resident glial cells (via metabolites such as SCFAs. The activation (or modulation) of lymphocytes and glial cells can lead to neuroinflammation or neurodegenerative disorders in the CNS. Figure made using (BioRender.com).

\section{INFLUENCE OF THE MICROBIOTA ON GLIAL CELLS IN CNS DISEASE}

Glial cells have been intensively studied in each MS, AD, and PD, and a role for the microbiome in modulating glial cell phenotype and function in these diseases is emerging.

\section{Microglia}

Microglia are CNS-resident macrophages serving key homeostatic and immune functions in the developing and adult CNS (1). Maternal microbiota can influence microglial maturation and function during both fetal development and in adulthood, as demonstrated in GF and antibiotic-treated mice (20, 21). Given the lack of evidence for a CNS microbiome, presumably microbiota-derived metabolites, such as short-chain fatty acids (SCFAs), directly or indirectly influence microglial phenotype (43). Microglia in GF mice are not fully mature, and interestingly, colonization with altered Schaedler's flora failed to rescue microglial defects whereas SCFA supplementation reversed the abnormal phenotype (21), indicating that the presence of SCFA-producing bacteria or a diverse microbiota are necessary for maturation.

In models of MS, antibiotic treatment prior to lysolecithin (LPC)-induced demyelination decreases microglia activation, indicated by the reduction in intralesional $\mathrm{P} 2 \mathrm{ry} 12^{\mathrm{lo}} \mathrm{Clec} 7 \mathrm{a}^{+}$ inflammatory cells as opposed to $\mathrm{P} 2 \mathrm{ry} 12^{+} \mathrm{Clec} 7 \mathrm{a}^{-}$homeostatic microglia. Microglial activation was also attenuated in GF mice given the demyelinating toxin cuprizone (44). Surprisingly, supplementing aged mice with probiotic VSL\#3 enhanced serum and fecal SCFAs, but had limited effect post-LPC administration (44). Indoxyl-3-sulfate (I3S), a product of microbial tryptophan metabolism, activates aryl hydrocarbon receptor (AHR) on microglia, augmenting TGF $\alpha$ production to limit astrocytic inflammation in EAE (45). Feeding whole milk promotes AHR ligand and SCFA production and ameliorates EAE in marmosets, although the effect is not attributed solely to microglia, but overall modulation of inflammation (46).

In $\alpha$-synuclein-overexpressing (ASO) mice that model PD, the presence of a gut microbiota promotes the aggregation of $\alpha$ synuclein in the caudoputamen and substantia nigra, resulting in microglial activation and motor dysfunction (47). GF ASO mice show significantly decreased levels of aggregated $\alpha$-synuclein and are protected from development of motor deficits. Re-colonization of GF mice with SPF microbiota reversed this rescue effect. Surprisingly, despite SCFAs being generally thought to be antiinflammatory, this study inculpates SCFAs in promoting aggregated $\alpha$-synuclein. SCFA supplementation to GF or antibiotic-treated ASO mice increased microglial activation and is sufficient to promote motor impairment. In addition, abundance of several SCFA-producing enzymes is increased in humanized mice that have received an FMT from Parkinson's disease donors.

Like other neurodegenerative diseases, an unhealthy microbiome is increasingly appreciated as a risk factor for $\mathrm{AD}$. 
Similar to PD, circulating levels of the SCFAs acetate and valerate were positively correlated with $\mathrm{A} \beta$ load in the brains of $\mathrm{AD}$ patients (48). Mice treated with probiotic bacteria exhibited ameliorated AD-like cognitive decline and decreased A $\beta$ aggregation (49). GF $5 \mathrm{x}$ Familial Alzheimer's disease (5xFAD) mice show a decreased $\mathrm{A} \beta$ load in the hippocampus compared to conventional $5 \mathrm{xFAD}$ mice, attributed to the uptake of $A \beta$ debris by microglia. A greater number of $\mathrm{Ibal}^{+}$cells were found in the hippocampus closely associated with $\mathrm{A} \beta$ plaques in GF $5 x \mathrm{xFD}$ mice, and a higher percentage of these plaque-associated $\mathrm{Iba}^{+}$cells were positive for methoxy-X-O4, an indicator of $\mathrm{A} \beta$ uptake (50).

\section{Astrocytes}

Astrocytes play diverse roles in the homeostatic brain that include providing trophic support to other CNS cells, regulation of synaptic activity, and controlling the blood-brain barrier (5154). Astrogliosis is also a feature of MS. Unsurprisingly, astrocytes play important roles in modulating neuroinflammation, as they can produce inflammatory cytokines and a host of chemokines that promote chemotaxis of other immune cells. In EAE, astrocytic inflammation is shown to be directly attenuated by I3S activation of AHR (45). Gut microbiota depletion by antibiotic treatment decreases levels of I3S and worsens EAE disease (55). AHRdeficient astrocytes increase expression of several proinflammatory chemokines and cytokines. Importantly, IFN $\beta$, a therapeutic used in some MS patients, works to limit CNS inflammation through this mechanism, as the anti-inflammatory effect of IFN $\beta$ is lost in AHR-deficient astrocytes (55).

\section{Oligodendrocytes}

Oligodendrocytes were previously thought to be quiescent, myelin-producing cells. However, increasing evidence shows that oligodendrocytes actively communicate with and provide metabolic support to neurons (56). Mature oligodendrocytes may also participate in remyelination and are active players during neurodegeneration (57). However, little is known about interactions between oligodendrocyte lineage cells and the gut microbiome. While treatment of mice with the probiotic VSL\#3 enhanced SCFA concentrations in feces and serum, there was no effect on remyelination in vivo following LPC-induced demyelination (44). Conversely, a separate study found that in ex vivo organotypic cerebellar slice cultures demyelinated by lysolecithin, the addition of the SCFA member butyrate enhanced both OPC numbers and mature oligodendrocyte numbers, indicating a positive effect on remyelination (58). In summary, the gut microbiota exerts effects not only on peripheral immune compartments, but also act on glial cells, with potential impacts on CNS disease processes.

\section{HOST FACTORS THAT INFLUENCE THE INTESTINAL MICROBIOME - A FOCUS ON IGA AND AGEING}

Many external factors influence the gut microbiome such as diet and pathogen exposure. In this section, we review host factors that shape the microbiome, focusing on IgA and ageing.

\section{Host Control of the Microbiota Through IgA}

Mature B cells primed in Peyer's patches can differentiate into IgA-producing PCs and home to the intestinal lamina propria $(41,59,60)$. The IgA produced by GALT PCs is typically dimeric, joined through the J-chain $(41,61)$. Secretory IgA is generated when dimeric IgA binds the polymeric-Ig-receptor (pIgR) on the basolateral surface of the intestinal epithelium, translocates through the epithelial cells, and is released into the lumen with the secretory component of the pIgR.

In mice, IgA both contributes to host control of microbiota and is responsive to gut microbiota changes. Mice monocolonized with Bacteroides thetaiotamicron harbor a reduced IgA repertoire restricted to a single clone against the capsular polysaccharide of the bacterium (62). Oral administration of Lactobacillus casei to mice resulted in an increase in $\operatorname{IgA}^{+}$cells in the small intestinal lamina propria (SILP) (63). Exposure of conventional mice to commensal Proteobacteria also resulted in increased serum IgA levels and induction of $\operatorname{IgA}^{+} \mathrm{PC}$ in the bone marrow (64). In contrast, some microbial communities can diminish IgA levels in the lumen due to their ability to degrade the secretory component (65). Even strain level differences in the microbiome can dictate IgA levels in the host (66). Conversely, the host IgA response can dictate the composition of the microbiome. Activation-induced cytidine deaminase-deficient mice (which fail to produce competent $\operatorname{IgA}$ ), exhibit an expansion of SFB in the small intestine which leads to isolated lymphoid follicle (ILF) hyperplasia and GC enlargement in secondary lymphoid tissues $(67,68)$. Restoration of IgA by heterogenetic parabiosis with wildtype mice reduced SFB populations, ILF protrusion, and spleen and lymph node size.

In humans, modest alterations in fecal microbiota composition are observed in subjects with selective IgAdeficiency (SIgAd) $(69,70)$. Compensatory sIgM in SIgAd subjects has a distinct bacterial binding pattern: an unclassified Enterobacteriaceae taxon heavily coated by $\operatorname{IgA}$ in healthy controls and by IgM in selective IgA-deficient subjects, was significantly more abundant in SIgAd subjects, demonstrating that IgA coating specifically restricts expansion of this taxon, and the same effect is not achievable by IgM.

Overall, these data indicate that in both mice and humans, a bi-directional relationship exists between host IgA and gut microbiota.

\section{Impact of Ageing on the Microbiome}

Growing evidence suggests that the gut microbiota has a critical impact on the ageing process and is a possible determinant of healthy ageing (71-73). Cross-sectional studies have examined alterations in the microbiota composition across the human lifespan $(74,75)$, demonstrating that taxonomical composition of gut microbiota appears to follow stepwise progression through life. Taxonomic shifts in the microbiota and decrease in microbial richness and diversity are observed in frail older individuals and associated with worse health outcomes compared to younger individuals (76-80). Relative abundance of Ruminococcaceae, Lachnospiraceae, and Bacteroidaceae families decrease with age, whereas an enrichment and/or higher prevalence of health-associated genera such as 
Akkermansia, Bifidobacterium, and Christensenellaceae, are maintained in longevity and extreme longevity (81). Indeed, centenarians tend to exhibit indicators of good health, and greater gut microbiota complexity (74). The relative abundance of pathobionts decreases and beneficial commensals, such as Akkermansia, are retained (82). Studies that stratify between elderly and centenarian status identify changes in taxa associated with extreme longevity including Odoribacter, Butyricimonas, Desulfovibrio, Bilophila, Oscillospira, and Akkermansia genera, and the Christensenellaceae and Barnesiellaceae families $(75,81)$.

Similar taxonomic and functional patterns that correlate with age and frailty in the mouse microbiome have been identified (83). In aged mice, the ratio of Firmicutes to Bacteroidetes increased $\sim 9$-fold compared to young mice, indicating dysbiosis, although this work was performed in commercially purchased mice rather than mice derived from the same dam (84). Introducing aged microbiota to young mice increased mortality following ischemic stroke, decreased behavioral outcomes, and increased cytokine levels. Conversely, altering the microbiota in aged mice to resemble that of young mice increased stroke survival and improved recovery (84). Changes in the gut microbiota in aged mice were also associated with increased gut permeability and elevations of peripheral inflammation $(85,86)$. Taken together, except for healthy centenarians who resist frailty, ageing is associated with an unhealthy microbiome.

\section{INFLUENCE OF AGEING AND IGA ON CNS DISEASE VIA THE MICROBIOME}

Multiple internal host factors impact the microbiome, including IgA and ageing. Here we speculate on how these two host factors may impact brain health and the trajectory of brain disease via the microbiome.

\section{$\lg \mathrm{A}$, the Microbiome, and CNS Disease}

Although $\operatorname{IgA}^{+}$ASCs can home to the dura mater during homeostasis (87), clonally expanded IgA are absent in steady state CNS and only appear during inflammation (88-91). During EAE, a significant reduction in $\operatorname{IgA}^{+}$ASCs is apparent in the SILP. Additionally, adoptively transferred gut-derived $\mathrm{IgA}^{+}$ ASCs were found in the CNS were reactive to mouse-derived gut bacteria and were shown to alleviate neuroinflammation by producing IL-10 at chronic stages of EAE. Over-abundance of $\mathrm{IgA}^{+}$ASCs was able to reduce GM-CSF production by T cells, an important cytokine that promotes neuroinflammation (18). Tritrichomonas musculis (T.mu) is a rodent commensal that promotes IgA production (92). EAE incidence and severity, as well as spinal cord inflammation and demyelination, are reduced in T. $m u^{+}$mice (18). T. $m u^{+}$mice also exhibited elevated serum and fecal IgA levels and increased frequencies of $\operatorname{IgA}^{+}$ASCs in the gut, bone marrow, and brain.

While the above highlights key findings from animal models, there is also early evidence suggesting the importance of the microbiota-driven IgA response in human disease. Bacteria identified by IgA-seq were differentially expressed in MS patients versus healthy controls (19). Stratified by disease activity, MS patients in relapse exhibited decreased percentages of IgA-bound gut bacteria in fecal samples compared to remitting patients, with corresponding elevation in CSF IgA. CNS-infiltrating $\operatorname{IgA}^{+} \mathrm{B}$ cells show specificity for gut microbial antigens, indicating the migration of IgA-producing cells from the gut during relapse. IgA is also elevated in cerebrospinal fluid of MS patients. Importantly, commensal-specific IgA ${ }^{+}$ASCs have been observed in inflammatory lesions of MS patients (19). This phenomenon may not be IgA-exclusive, however, as IgG in MS patient CSF has been found to be reactive against MSassociated gut bacterial lysate (93). The implications of these bacteria-reactive IgG in disease have yet to be fully elucidated.

Lastly, while $\operatorname{IgA}^{+}$ASC have been now described in the inflamed EAE and MS CNS, it is now appreciated that these cells play an important role in homeostasis. Specifically, intestinal commensal specific $\operatorname{IgA}^{+}$ASC have been detected in the leptomeninges of healthy mice and humans but are absent in GF mice (87). These cells likely maintain barrier integrity near the dural sinuses; however, it is possible they may also contribute to quiescence within the CNS.

In summary, in addition to its well appreciated role in shaping the microbiome, IgA-producing ASC likewise play important roles in the healthy and MS/EAE CNS. The role for these cells in $\mathrm{PD}$ and $\mathrm{AD}$ is not yet understood.

\section{Ageing, the Microbiome, and CNS Disease}

Ageing is the predominant risk factor for neurodegenerative diseases (94), yet in spite of the known role ageing has on the microbiome, the connection between ageing, the microbiome and CNS disease has barely been explored.

It is well established that microglia are affected during ageing. Ageing results in decreased number, uneven distribution, lower motility, and fewer ramifications, as well as impairment in phagocytosis and injury responses (2, 95-98). Senescent microglia increase pro-inflammatory cytokine production (3). An altered microglia morphology and reduced arborization have been observed in the human brain during ageing and age-related diseases such as AD (95). This dystrophic morphology is associated with impaired spatial learning (3).

Age-related changes in the gut microbiome may have a direct impact on microglial function within the CNS. In fact, reduced complexity of microbiota, a feature of ageing, leads to defects in microglia maturation and function (21). Recent work demonstrated that FMT from aged donor mice into young recipients impairs spatial learning and memory in young recipients (4). Conversely, FMT from young donor mice into aged recipients can rejuvenate age-associated CNS metabolic, transcriptomic, and behavioral changes (5). Aged into young FMT induced an altered expression of proteins involved in synaptic plasticity and neurotransmission in the hippocampus, an area of the CNS known to be affected by the ageing process. A strong reduction of bacteria associated with SCFA production (Lachnospiraceae, Faecalibaculum, and Ruminococcaceae) and 
disorders of the CNS (Prevotellaceae and Ruminococcaceae) was also reported (4). Interestingly, microglia of the hippocampus acquired an ageing-like phenotype following FMT. Of therapeutic relevance, this age-associated phenotype can be reversed by re-introducing live and complex microbiota or microbial metabolites, such as SCFAs (6).

The gut microbiota similarly affects astrocytes in both ageing and age-associated neurodegenerative diseases (7). Ageing can alter the normal function of astrocytes which reduces their ability to properly maintain a healthy CNS environment (8). Astrocyte transcriptomes from multiple mouse brain regions have revealed that ageing upregulates genes that eliminate synapses and induces a reactive astrocyte gene signature (9). Therefore, aged astrocytes may promote synapse elimination and neuronal damage, contributing to ageing-associated cognitive decline. Morphological changes in astrocytes have also been documented in the aged CNS (10-12). Aged astrocytes increase cytokine production, notably CXCL10 (13) that attracts peripheral immune cells and promotes $\mathrm{T}$ cell adhesion to endothelial cells (14). CXCR3, which is the CXCL10 receptor, is expressed in microglia, suggesting that astrocytes and microglia communicate during ageing $(22,45)$.

\section{REFERENCES}

1. Nimmerjahn A, Kirchhoff F, Helmchen F. Resting Microglial Cells Are Highly Dynamic Surveillants of Brain Parenchyma In Vivo. Sci (New York NY) (2005) 308(5726):1314-8. doi: 10.1126/science. 1110647

2. Zoller T, Attaai A, Potru PS, Russ T, Spittau B. Aged Mouse Cortical Microglia Display an Activation Profile Suggesting Immunotolerogenic Functions. Int J Mol Sci (2018) 19(3):706. doi: 10.3390/ijms19030706

3. Niraula A, Sheridan JF, Godbout JP. Microglia Priming With Aging and Stress. Neuropsychopharmacology (2017) 42(1):318-33. doi: 10.1038/ npp.2016.185

4. D’Amato A, Di Cesare Mannelli L, Lucarini E, Man AL, Le Gall G, Branca JJV, et al. Faecal Microbiota Transplant From Aged Donor Mice Affects Spatial Learning and Memory via Modulating Hippocampal Synaptic Plasticity- and Neurotransmission-Related Proteins in Young Recipients. Microbiome (2020) 8(1):140. doi: 10.1186/s40168-020-00914-w

5. Boehme M, Guzzetta KE, Bastiaanssen TFS, van de Wouw M, Moloney GM, Gual-Grau A, et al. Microbiota From Young Mice Counteracts Selective AgeAssociated Behavioral Deficits. Nat Aging (2021) 1(8):666-76. doi: 10.1038/ s43587-021-00093-9

6. Shen P, Roch T, Lampropoulou V, O'Connor RA, Stervbo U, Hilgenberg E, et al. IL-35-Producing B Cells Are Critical Regulators of Immunity During Autoimmune and Infectious Diseases. Nature (2014) 507(7492):366-70. doi: 10.1038/nature12979

7. Meldolesi J. Astrocytes: News About Brain Health and Diseases. Biomedicines (2020) 8(10):394. doi: 10.3390/biomedicines 8100394

8. Palmer AL, Ousman SS. Astrocytes and Aging. Front Aging Neurosci (2018) 10:337. doi: 10.3389/fnagi.2018.00337

9. Boisvert MM, Erikson GA, Shokhirev MN, Allen NJ. The Aging Astrocyte Transcriptome From Multiple Regions of the Mouse Brain. Cell Rep (2018) 22 (1):269-85. doi: 10.1016/j.celrep.2017.12.039

10. Jyothi HJ, Vidyadhara DJ, Mahadevan A, Philip M, Parmar SK, Manohari SG, et al. Aging Causes Morphological Alterations in Astrocytes and Microglia in Human Substantia Nigra Pars Compacta. Neurobiol Aging (2015) 36 (12):3321-33. doi: 10.1016/j.neurobiolaging.2015.08.024

11. Amenta F, Bronzetti E, Sabbatini M, Vega JA. Astrocyte Changes in Aging Cerebral Cortex and Hippocampus: A Quantitative Immunohistochemical Study. Microsc Res Tech (1998) 43(1):29-33. doi: 10.1002/(SICI)1097-0029 (19981001)43:1<29::AID-JEMT5>3.0.CO;2-H

\section{CONCLUSIONS}

Chronic, complex diseases of the CNS develop over years. Animal studies conducted under controlled conditions in short periods miss two large confounders in these diseases - time (ageing) and the microbiota-IgA axis, with age-associated microbiota alterations further complicating this relationship. These are important considerations for animal modelling, given the considerable variability in microbiota composition and gut luminal IgA levels between vivaria (65). In summary, we propose that host factors such as age and intestinal IgA are key determinants in how the microbiome impacts lymphocyte and glial cell phenotype/function in the context of MS, AD and PD (Figure 2).

\section{AUTHOR CONTRIBUTIONS}

$\mathrm{AP}, \mathrm{DL}, \mathrm{BI}$, and IN all contributed to the writing of this manuscript. AP, DL, and JG contributed to the editing of the text and generation of all figures. All authors contributed to the article and approved the submitted version.

12. Robillard KN, Lee KM, Chiu KB, MacLean AG. Glial Cell Morphological and Density Changes Through the Lifespan of Rhesus Macaques. Brain Behav Immun (2016) 55:60-9. doi: 10.1016/j.bbi.2016.01.006

13. Clarke LE, Liddelow SA, Chakraborty C, Munch AE, Heiman M, Barres BA. Normal Aging Induces A1-Like Astrocyte Reactivity. Proc Natl Acad Sci USA (2018) 115(8):E1896-905. doi: 10.1073/pnas.1800165115

14. Sorensen EW, Lian J, Ozga AJ, Miyabe Y, Ji SW, Bromley SK, et al. CXCL10 Stabilizes T Cell-Brain Endothelial Cell Adhesion Leading to the Induction of Cerebral Malaria. JCI Insight (2018) 3(8):e98911. doi: 10.1172/jci.insight.98911

15. Miyake S, Kim S, Suda W, Oshima K, Nakamura M, Matsuoka T, et al. Dysbiosis in the Gut Microbiota of Patients With Multiple Sclerosis, With a Striking Depletion of Species Belonging to Clostridia XIVa and IV Clusters. PloS One (2015) 10(9):e0137429. doi: 10.1371/journal.pone.0137429

16. Johanson DM, Goertz JE, Marin IA, Costello J, Overall CC, Gaultier A. Experimental Autoimmune Encephalomyelitis Is Associated With Changes of the Microbiota Composition in the Gastrointestinal Tract. Sci Rep (2020) 10 (1):15183. doi: 10.1038/s41598-020-72197-y

17. Ochoa-Repáraz J, Mielcarz DW, Wang Y, Begum-Haque S, Dasgupta S, Kasper DL, et al. A Polysaccharide From the Human Commensal Bacteroides Fragilis Protects Against CNS Demyelinating Disease. Mucosal Immunol (2010) 3(5):487-95. doi: 10.1038/mi.2010.29

18. Rojas OL, Probstel AK, Porfilio EA, Wang AA, Charabati M, Sun T, et al. Recirculating Intestinal IgA-Producing Cells Regulate Neuroinflammation via IL-10. Cell (2019) 176(3):610-24.e18. doi: 10.1016/j.cell.2018.11.035

19. Pröbstel A-K, Zhou X, Baumann R, Wischnewski S, Kutza M, Rojas OL, et al. Gut Microbiota-Specific IgA+ B Cells Traffic to the CNS in Active Multiple Sclerosis. Sci Immunol (2020) 5(53):eabc7191. doi: 10.1126/sciimmunol.abc7191

20. Matcovitch-Natan O, Winter DR, Giladi A, Vargas Aguilar S, Spinrad A, Sarrazin S, et al. Microglia Development Follows a Stepwise Program to Regulate Brain Homeostasis. Sci (New York NY) (2016) 353(6301):aad8670. doi: $10.1126 /$ science.aad8670

21. Erny D, Hrabe de Angelis AL, Jaitin D, Wieghofer P, Staszewski O, David E, et al. Host Microbiota Constantly Control Maturation and Function of Microglia in the CNS. Nat Neurosci (2015) 18(7):965-77. doi: 10.1038/nn.4030

22. Liddelow SA, Barres BA. Reactive Astrocytes: Production, Function, and Therapeutic Potential. Immunity (2017) 46(6):957-67. doi: 10.1016/ j.immuni.2017.06.006

23. Scheperjans F, Aho V, Pereira PA, Koskinen K, Paulin L, Pekkonen E, et al. Gut Microbiota Are Related to Parkinson's Disease and Clinical Phenotype. Mov Disord (2015) 30(3):350-8. doi: 10.1002/mds.26069 
24. Human Microbiome Jumpstart Reference Strains Consortium, Nelson KE, Weinstock GM, Highlander SK, Worley KC, Creasy HH, et al. A Catalog of Reference Genomes From the Human Microbiome. Sci (New York NY) (2010) 328(5981):994-9. doi: 10.1126/science.1183605

25. Deschasaux M, Bouter KE, Prodan A, Levin E, Groen AK, Herrema H, et al. Depicting the Composition of Gut Microbiota in a Population With Varied Ethnic Origins But Shared Geography. Nat Med (2018) 24(10):1526-31. doi: 10.1038/s41591-018-0160-1

26. David LA, Maurice CF, Carmody RN, Gootenberg DB, Button JE, Wolfe BE, et al. Diet Rapidly and Reproducibly Alters the Human Gut Microbiome. Nature (2014) 505(7484):559-63. doi: 10.1038/nature12820

27. O’Keefe SJD, Li JV, Lahti L, Ou J, Carbonero F, Mohammed K, et al. Fat, Fibre and Cancer Risk in African Americans and Rural Africans. Nat Commun (2015) 6(1):6342. doi: 10.1038/ncomms7342

28. Vangay P, Johnson AJ, Ward TL, Al-Ghalith GA, Shields-Cutler RR, Hillmann BM, et al. US Immigration Westernizes the Human Gut Microbiome. Cell (2018) 175(4):962-72.e10. doi: 10.1016/j.cell.2018.10.029

29. Perez-Munoz ME, Sugden S, Harmsen HJM, t Hart BA, Laman JD, Walter J. Nutritional and Ecological Perspectives of the Interrelationships Between Diet and the Gut Microbiome in Multiple Sclerosis: Insights From Marmosets. iScience (2021) 24(7):102709. doi: 10.1016/j.isci.2021.102709

30. Groot HE, van de Vegte YJ, Verweij N, Lipsic E, Karper JC, van der Harst P. Human Genetic Determinants of the Gut Microbiome and Their Associations With Health and Disease: A Phenome-Wide Association Study. Sci Rep (2020) 10(1):14771. doi: 10.1038/s41598-020-70724-5

31. Colpitts SL, Kasper EJ, Keever A, Liljenberg C, Kirby T, Magori K, et al. A Bidirectional Association Between the Gut Microbiota and CNS Disease in a Biphasic Murine Model of Multiple Sclerosis. Gut Microbes (2017) 8(6):56173. doi: 10.1080/19490976.2017.1353843

32. Jangi S, Gandhi R, Cox LM, Li N, von Glehn F, Yan R, et al. Alterations of the Human Gut Microbiome in Multiple Sclerosis. Nat Commun (2016) 7:12015. doi: $10.1038 /$ ncomms 12015

33. Moles L, Egimendia A, Osorio-Querejeta I, Iparraguirre L, Alberro A, Suárez J, et al. Gut Microbiota Changes in Experimental Autoimmune Encephalomyelitis and Cuprizone Mice Models. ACS Chem Neurosci (2021) 12(5):893-905. doi: 10.1021/acschemneuro.0c00695

34. Tremlett H, Bauer KC, Appel-Cresswell S, Finlay BB, Waubant E. The Gut Microbiome in Human Neurological Disease: A Review. Ann Neurol (2017) 81(3):369-82. doi: 10.1002/ana.24901

35. Lee YK, Menezes JS, Umesaki Y, Mazmanian SK. Proinflammatory T-Cell Responses to Gut Microbiota Promote Experimental Autoimmune Encephalomyelitis. Proc Natl Acad Sci USA (2011) 108(Suppl 1):4615-22. doi: 10.1073/pnas.1000082107

36. Ivanov II, Atarashi K, Manel N, Brodie EL, Shima T, Karaoz U, et al. Induction of Intestinal Th17 Cells by Segmented Filamentous Bacteria. Cell (2009) 139 (3):485-98. doi: 10.1016/j.cell.2009.09.033

37. Berer K, Gerdes LA, Cekanaviciute E, Jia X, Xiao L, Xia Z, et al. Gut Microbiota From Multiple Sclerosis Patients Enables Spontaneous Autoimmune Encephalomyelitis in Mice. Proc Natl Acad Sci USA (2017) 114(40):10719-24. doi: 10.1073/pnas.1711233114

38. Cekanaviciute E, Yoo BB, Runia TF, Debelius JW, Singh S, Nelson CA, et al. Gut Bacteria From Multiple Sclerosis Patients Modulate Human T Cells and Exacerbate Symptoms in Mouse Models. Proc Natl Acad Sci USA (2017) 114 (40):10713-8. doi: 10.1073/pnas.1711235114

39. De Silva NS, Klein U. Dynamics of B Cells in Germinal Centres. Nat Rev Immunol (2015) 15(3):137-48. doi: 10.1038/nri3804

40. Nutt SL, Hodgkin PD, Tarlinton DM, Corcoran LM. The Generation of Antibody-Secreting Plasma Cells. Nat Rev Immunol (2015) 15(3):160-71. doi: 10.1038/nri3795

41. Brandtzaeg P, Johansen FE. Mucosal B Cells: Phenotypic Characteristics, Transcriptional Regulation, and Homing Properties. Immunol Rev (2005) 206:32-63. doi: 10.1111/j.0105-2896.2005.00283.x

42. Ochoa-Repáraz J, Mielcarz DW, Haque-Begum S, Kasper LH. Induction of a Regulatory B Cell Population in Experimental Allergic Encephalomyelitis by Alteration of the Gut Commensal Microflora. Gut Microbes (2010) 1(2):1038. doi: 10.4161/gmic.1.2.11515

43. Parada Venegas D, de la Fuente MK, Landskron G, Gonzalez MJ, Quera R, Dijkstra G, et al. Short Chain Fatty Acids (SCFAs)-Mediated Gut Epithelial and Immune Regulation and Its Relevance for Inflammatory Bowel Diseases. Front Immunol (2019) 10:277. doi: 10.3389/fimmu.2019.00277

44. McMurran CE, Guzman de la Fuente A, Penalva R, Ben Menachem-Zidon O, Dombrowski Y, Falconer J, et al. The Microbiota Regulates Murine Inflammatory Responses to Toxin-Induced CNS Demyelination But Has Minimal Impact on Remyelination. Proc Natl Acad Sci USA (2019) 116 (50):25311-21. doi: 10.1073/pnas.1905787116

45. Rothhammer V, Borucki DM, Tjon EC, Takenaka MC, Chao CC, ArduraFabregat A, et al. Microglial Control of Astrocytes in Response to Microbial Metabolites. Nature (2018) 557(7707):724-8. doi: 10.1038/s41586-018-0119-x

46. Kap YS, Bus-Spoor C, van Driel N, Dubbelaar ML, Grit C, Kooistra SM, et al. Targeted Diet Modification Reduces Multiple Sclerosis-Like Disease in Adult Marmoset Monkeys From an Outbred Colony. J Immunol (2018) 201 (11):3229. doi: 10.4049/jimmunol.1800822

47. Sampson TR, Debelius JW, Thron T, Janssen S, Shastri GG, Ilhan ZE, et al. Gut Microbiota Regulate Motor Deficits and Neuroinflammation in a Model of Parkinson's Disease. Cell (2016) 167(6):1469-80.e12. doi: 10.1016/j.cell. 2016.11.018

48. Marizzoni M, Cattaneo A, Mirabelli P, Festari C, Lopizzo N, Nicolosi V, et al. Short-Chain Fatty Acids and Lipopolysaccharide as Mediators Between Gut Dysbiosis and Amyloid Pathology in Alzheimer's Disease. J Alzheimers Dis (2020) 78(2):683-97. doi: 10.3233/JAD-200306

49. Bonfili L, Cecarini V, Berardi S, Scarpona S, Suchodolski JS, Nasuti C, et al. Microbiota Modulation Counteracts Alzheimer's Disease Progression Influencing Neuronal Proteolysis and Gut Hormones Plasma Levels. Sci Rep (2017) 7(1):2426. doi: 10.1038/s41598-017-02587-2

50. Mezo C, Dokalis N, Mossad O, Staszewski O, Neuber J, Yilmaz B, et al. Different Effects of Constitutive and Induced Microbiota Modulation on Microglia in a Mouse Model of Alzheimer's Disease. Acta Neuropathol Commun (2020) 8(1):119. doi: 10.1186/s40478-020-00988-5

51. Abbott NJ, Ronnback L, Hansson E. Astrocyte-Endothelial Interactions at the Blood-Brain Barrier. Nat Rev Neurosci (2006) 7(1):41-53. doi: 10.1038/nrn1824

52. Perea G, Araque A. Properties of Synaptically Evoked Astrocyte Calcium Signal Reveal Synaptic Information Processing by Astrocytes. J Neurosci (2005) 25(9):2192-203. doi: 10.1523/JNEUROSCI.3965-04.2005

53. Bezzi P, Gundersen V, Galbete JL, Seifert G, Steinhauser C, Pilati E, et al. Astrocytes Contain a Vesicular Compartment That Is Competent for Regulated Exocytosis of Glutamate. Nat Neurosci (2004) 7(6):613-20. doi: $10.1038 / \mathrm{nn} 1246$

54. Sofroniew MV, Vinters HV. Astrocytes: Biology and Pathology. Acta Neuropathol (2010) 119(1):7-35. doi: 10.1007/s00401-009-0619-8

55. Rothhammer V, Mascanfroni ID, Bunse L, Takenaka MC, Kenison JE, Mayo L, et al. Type I Interferons and Microbial Metabolites of Tryptophan Modulate Astrocyte Activity and Central Nervous System Inflammation via the Aryl Hydrocarbon Receptor. Nat Med (2016) 22(6):586-97. doi: 10.1038/nm.4106

56. Krasnow AM, Ford MC, Valdivia LE, Wilson SW, Attwell D. Regulation of Developing Myelin Sheath Elongation by Oligodendrocyte Calcium Transients In Vivo. Nat Neurosci (2018) 21(1):24-8. doi: 10.1038/s41593017-0031-y

57. Duncan ID, Radcliff AB, Heidari M, Kidd G, August BK, Wierenga LA. The Adult Oligodendrocyte Can Participate in Remyelination. Proc Natl Acad Sci USA (2018) 115(50):E11807-16. doi: 10.1073/pnas.1808064115

58. Chen T, Noto D, Hoshino Y, Mizuno M, Miyake S. Butyrate Suppresses Demyelination and Enhances Remyelination. J Neuroinflamm (2019) 16 (1):165. doi: 10.1186/s12974-019-1552-y

59. Fagarasan S, Kawamoto S, Kanagawa O, Suzuki K. Adaptive Immune Regulation in the Gut: T Cell-Dependent and T Cell-Independent IgA Synthesis. Annu Rev Immunol (2010) 28:243-73. doi: 10.1146/annurevimmunol-030409-101314

60. Wang AA, Gommerman JL, Rojas OL. Plasma Cells: From Cytokine Production to Regulation in Experimental Autoimmune Encephalomyelitis. J Mol Biol (2020) 8:166655. doi: 10.1016/j.jmb.2020.09.014

61. Chung JB, Silverman M, Monroe JG. Transitional B Cells: Step by Step Towards Immune Competence. Trends Immunol (2003) 24(6):343-9. doi: 10.1016/S1471-4906(03)00119-4

62. Peterson DA, McNulty NP, Guruge JL, Gordon JI. IgA Response to Symbiotic Bacteria as a Mediator of Gut Homeostasis. Cell Host Microbe (2007) 2 (5):328-39. doi: 10.1016/j.chom.2007.09.013 
63. Galdeano CM, Perdigon G. The Probiotic Bacterium Lactobacillus Casei Induces Activation of the Gut Mucosal Immune System Through Innate Immunity. Clin Vaccine Immunol (2006) 13(2):219-26. doi: 10.1128/CVI.13.2.219-226.2006

64. Wilmore JR, Gaudette BT, Gomez Atria D, Hashemi T, Jones DD, Gardner CA, et al. Commensal Microbes Induce Serum IgA Responses That Protect Against Polymicrobial Sepsis. Cell Host Microbe (2018) 23(3):302-11.e3. doi: 10.1016/j.chom.2018.01.005

65. Moon C, Baldridge MT, Wallace MA, Carey-Ann D, Burnham, Virgin HW, et al. Vertically Transmitted Faecal IgA Levels Determine Extra-Chromosomal Phenotypic Variation. Nature (2015) 521(7550):90-3. doi: 10.1038/nature14139

66. Yang C, Mogno I, Contijoch EJ, Borgerding JN, Aggarwala V, Li Z, et al. Fecal IgA Levels Are Determined by Strain-Level Differences in Bacteroides Ovatus and Are Modifiable by Gut Microbiota Manipulation. Cell Host Microbe (2020) 27(3):467-75.e6. doi: 10.1016/j.chom.2020.01.016

67. Suzuki K, Meek B, Doi Y, Muramatsu M, Chiba T, Honjo T, et al. Aberrant Expansion of Segmented Filamentous Bacteria in IgA-Deficient Gut. Proc Natl Acad Sci USA (2004) 101(7):1981-6. doi: 10.1073/pnas.0307317101

68. Fagarasan S, Muramatsu M, Suzuki K, Nagaoka H, Hiai H, Honjo T. Critical Roles of Activation-Induced Cytidine Deaminase in the Homeostasis of Gut Flora. Sci (New York NY) (2002) 298(5597):1424-7. doi: 10.1126/science.1077336

69. Fadlallah J, El Kafsi H, Sterlin D, Juste C, Parizot C, Dorgham K, et al. Microbial Ecology Perturbation in Human IgA Deficiency. Sci Transl Med (2018) 10(439):eaan1217. doi: 10.1126/scitranslmed.aan1217

70. Catanzaro JR, Strauss JD, Bielecka A, Porto AF, Lobo FM, Urban A, et al. IgADeficient Humans Exhibit Gut Microbiota Dysbiosis Despite Secretion of Compensatory IgM. Sci Rep (2019) 9(1):13574. doi: 10.1038/s41598-019-49923-2

71. Claesson MJ, Jeffery IB, Conde S, Power SE, O'Connor EM, Cusack S, et al. Gut Microbiota Composition Correlates With Diet and Health in the Elderly. Nature (2012) 488(7410):178-84. doi: 10.1038/nature11319

72. Candela M, Biagi E, Brigidi P, O’Toole PW, De Vos WM. Maintenance of a Healthy Trajectory of the Intestinal Microbiome During Aging: A Dietary Approach. Mech Ageing Dev (2014) 136-137:70-5. doi: 10.1016/j.mad. 2013.12.004

73. Heintz C, Mair W. You Are What You Host: Microbiome Modulation of the Aging Process. Cell (2014) 156(3):408-11. doi: 10.1016/j.cell.2014.01.025

74. Odamaki T, Kato K, Sugahara H, Hashikura N, Takahashi S, J-z X, et al. AgeRelated Changes in Gut Microbiota Composition From Newborn to Centenarian: A Cross-Sectional Study. BMC Microbiol (2016) 16(1):90. doi: 10.1186/s12866-016-0708-5

75. Xu C, Zhu H, Qiu P. Aging Progression of Human Gut Microbiota. BMC Microbiol (2019) 19(1):236. doi: 10.1186/s12866-019-1616-2

76. Claesson MJ, Cusack S, O’Sullivan O, Greene-Diniz R, de Weerd H, Flannery E, et al. Composition, Variability, and Temporal Stability of the Intestinal Microbiota of the Elderly. Proc Natl Acad Sci USA (2011) 108(Suppl 1):458691. doi: 10.1073/pnas.1000097107

77. Jackson MA, Jeffery IB, Beaumont M, Bell JT, Clark AG, Ley RE, et al. Signatures of Early Frailty in the Gut Microbiota. Genome Med (2016) 8(1):8. doi: 10.1186/s13073-016-0275-2

78. O’Toole PW, Jeffery IB. Gut Microbiota and Aging. Sci (New York NY) (2015) 350(6265):1214-5. doi: 10.1126/science.aac8469

79. O'Toole PW, Jeffery IB. Microbiome-Health Interactions in Older People. Cell Mol Life Sci (2018) 75(1):119-28. doi: 10.1007/s00018-017-2673-z

80. Zapata HJ, Quagliarello VJ. The Microbiota and Microbiome in Aging: Potential Implications in Health and Age-Related Diseases. J Am Geriatr Soc (2015) 63(4):776-81. doi: 10.1111/jgs.13310

81. Biagi E, Franceschi C, Rampelli S, Severgnini M, Ostan R, Turroni S, et al. Gut Microbiota and Extreme Longevity. Curr Biology: CB (2016) 26(11):1480-5. doi: 10.1016/j.cub.2016.04.016

82. Ragonnaud E, Biragyn A. Gut Microbiota as the Key Controllers of "Healthy" Aging of Elderly People. Immun Ageing (2021) 18(1):2. doi: 10.1186/s12979020-00213-w

83. Langille MG, Meehan CJ, Koenig JE, Dhanani AS, Rose RA, Howlett SE, et al. Microbial Shifts in the Aging Mouse Gut. Microbiome (2014) 2(1):50. doi: 10.1186/s40168-014-0050-9

84. Spychala MS, Venna VR, Jandzinski M, Doran SJ, Durgan DJ, Ganesh BP, et al. Age-Related Changes in the Gut Microbiota Influence Systemic Inflammation and Stroke Outcome. Ann Neurol (2018) 84(1):23-36. doi: 10.1002/ana. 25250
85. Scott KA, Ida M, Peterson VL, Prenderville JA, Moloney GM, Izumo T, et al. Revisiting Metchnikoff: Age-Related Alterations in Microbiota-Gut-Brain Axis in the Mouse. Brain Behav Immun (2017) 65:20-32. doi: 10.1016/ j.bbi.2017.02.004

86. Thevaranjan N, Puchta A, Schulz C, Naidoo A, Szamosi JC, Verschoor CP, et al. Age-Associated Microbial Dysbiosis Promotes Intestinal Permeability, Systemic Inflammation, and Macrophage Dysfunction. Cell Host Microbe (2017) 21(4):455-66.e4. doi: 10.1016/j.chom.2017.03.002

87. Fitzpatrick Z, Frazer G, Ferro A, Clare S, Bouladoux N, Ferdinand J, et al. GutEducated IgA Plasma Cells Defend the Meningeal Venous Sinuses. Nature (2020) 587(7834):472-6. doi: 10.1038/s41586-020-2886-4

88. Olsson JE, Link H. Immunoglobulin Abnormalities in Multiple Sclerosis. Relation to Clinical Parameters: Exacerbations and Remissions. Arch Neurol (1973) 28(6):392-9. doi: 10.1001/archneur.1973.00490240052009

89. Link H, Müller R. Immunoglobulins in Multiple Sclerosis and Infections of the Nervous System. Arch Neurol (1971) 25(4):326-44. doi: 10.1001/ archneur.1971.00490040052007

90. Omura S, Sato F, Park AM, Fujita M, Khadka S, Nakamura Y, et al. Bioinformatics Analysis of Gut Microbiota and CNS Transcriptome in Virus-Induced Acute Myelitis and Chronic Inflammatory Demyelination; Potential Association of Distinct Bacteria With CNS IgA Upregulation. Front Immunol (2020) 11:1138. doi: 10.3389/fimmu.2020.01138

91. Isho B, Florescu A, Wang AA, Gommerman JL. Fantastic IgA Plasma Cells and Where to Find Them. Immunol Rev (2021) 303:119-37. doi: 10.1111/ imr. 12980

92. Chudnovskiy A, Mortha A, Kana V, Kennard A, Ramirez JD, Rahman A, et al. Host-Protozoan Interactions Protect From Mucosal Infections Through Activation of the Inflammasome. Cell (2016) 167(2):444-56.e14. doi: 10.1016/j.cell.2016.08.076

93. Eckman E, Laman JD, Fischer KF, Lopansri B, Martins TB, Hill HR, et al. Spinal Fluid IgG Antibodies From Patients With Demyelinating Diseases Bind Multiple Sclerosis-Associated Bacteria. J Mol Med (Berl) (2021), 1-13. doi: 10.1007/s00109-021-02085-Z

94. Hou Y, Dan X, Babbar M, Wei Y, Hasselbalch SG, Croteau DL, et al. Ageing as a Risk Factor for Neurodegenerative Disease. Nat Rev Neurol (2019) 15 (10):565-81. doi: 10.1038/s41582-019-0244-7

95. Davies DS, Ma J, Jegathees T, Goldsbury C. Microglia Show Altered Morphology and Reduced Arborization in Human Brain During Aging and Alzheimer's Disease. Brain Pathol (2017) 27(6):795-808. doi: 10.1111/ bpa. 12456

96. Soreq LConsortium UKBE and North American Brain Expression C, , Rose J, Soreq E, Hardy J, et al. Major Shifts in Glial Regional Identity Are a Transcriptional Hallmark of Human Brain Aging. Cell Rep (2017) 18 (2):557-70. doi: 10.1016/j.celrep.2016.12.011

97. Streit WJ. Microglial Senescence: Does the Brain's Immune System Have an Expiration Date? Trends Neurosci (2006) 29(9):506-10. doi: 10.1016/ j.tins.2006.07.001

98. Wasserman JK, Yang H, Schlichter LC. Glial Responses, Neuron Death and Lesion Resolution After Intracerebral Hemorrhage in Young vs. Aged Rats. Eur J Neurosci (2008) 28(7):1316-28. doi: 10.1111/j.1460-9568. 2008.06442.x

Conflict of Interest: The authors declare that the research was conducted in the absence of any commercial or financial relationships that could be construed as a potential conflict of interest.

Publisher's Note: All claims expressed in this article are solely those of the authors and do not necessarily represent those of their affiliated organizations, or those of the publisher, the editors and the reviewers. Any product that may be evaluated in this article, or claim that may be made by its manufacturer, is not guaranteed or endorsed by the publisher.

Copyright (c) $2021 \mathrm{Pu}$, Lee, Isho, Naouar and Gommerman. This is an open-access article distributed under the terms of the Creative Commons Attribution License (CC BY). The use, distribution or reproduction in other forums is permitted, provided the original author(s) and the copyright owner(s) are credited and that the original publication in this journal is cited, in accordance with accepted academic practice. No use, distribution or reproduction is permitted which does not comply with these terms. 